\title{
On Hyers-Ulam Mittag-Leffler stability of discrete fractional Duffing equation with application on inverted pendulum
}

\author{
A.G.M. Selvam', D. Baleanu²,3 J. Alzabut ${ }^{4 *}$ (D, D. Vignesh and S. Abbas ${ }^{5}$
}

\section{"Correspondence:}

jalzabut@psu.edu.sa

${ }^{4}$ Department of Mathematics and

General Sciences, Prince Sultan

University, Riyadh-11586, Saudi

Arabia

Full list of author information is

available at the end of the article

\begin{abstract}
A human being standing upright with his feet as the pivot is the most popular example of the stabilized inverted pendulum. Achieving stability of the inverted pendulum has become common challenge for engineers. In this paper, we consider an initial value discrete fractional Duffing equation with forcing term. We establish the existence, Hyers-Ulam stability, and Hyers-Ulam Mittag-Leffler stability of solutions for the equation. We consider the inverted pendulum modeled by Duffing equation as an example. The values are tabulated and simulated to show the consistency with theoretical findings.
\end{abstract}

MSC: 26A33; 39A30

Keywords: Fractional Duffing equation; Mittag-Leffler function; Hyers-Ulam stability; Inverted pendulum

\section{Introduction}

The understanding of the real-world problems by replicating into mathematical models proves to be an effective tool. Analyzing the developed model thus provides a wide insight into the considered phenomena. In [1], Rayleigh introduced a nonlinear damping function in a second-order oscillator equation. Extensive study of this equation using vacuum-tube circuits for analogue simulation was carried out by Van der Pol [2]. Besides, a model of heart beat was constructed using an electrical circuit with coupled relaxation oscillators and simulations of normal heart beat and of certain disorders were convincingly obtained by Van der Pol and Van der Mark [3]. Certain damped and driven oscillators are modeled by the Duffing equation, a second-order differential equation with cubic nonlinearity named after Georg Duffing [4]. The motion of a damped oscillator described by the equation has more complex potential than simple harmonic motion. This equation is used to illustrate the motion of a mass attached to a nonlinear spring and a linear damper. The Duffing equation is given by

$$
\ddot{x}+\theta \dot{x}+\delta x+\eta x^{3}=\gamma \sin (\omega t)
$$

(c) The Author(s) 2020. This article is licensed under a Creative Commons Attribution 4.0 International License, which permits use, sharing, adaptation, distribution and reproduction in any medium or format, as long as you give appropriate credit to the original author(s) and the source, provide a link to the Creative Commons licence, and indicate if changes were made. The images or other third party material in this article are included in the article's Creative Commons licence, unless indicated otherwise in a credit line to the material. If material is not included in the article's Creative Commons licence and your intended use is not permitted by statutory regulation or exceeds the permitted use, you will need to obtain permission directly from the copyright holder. To view a copy of this licence, visit http://creativecommons.org/licenses/by/4.0/. 


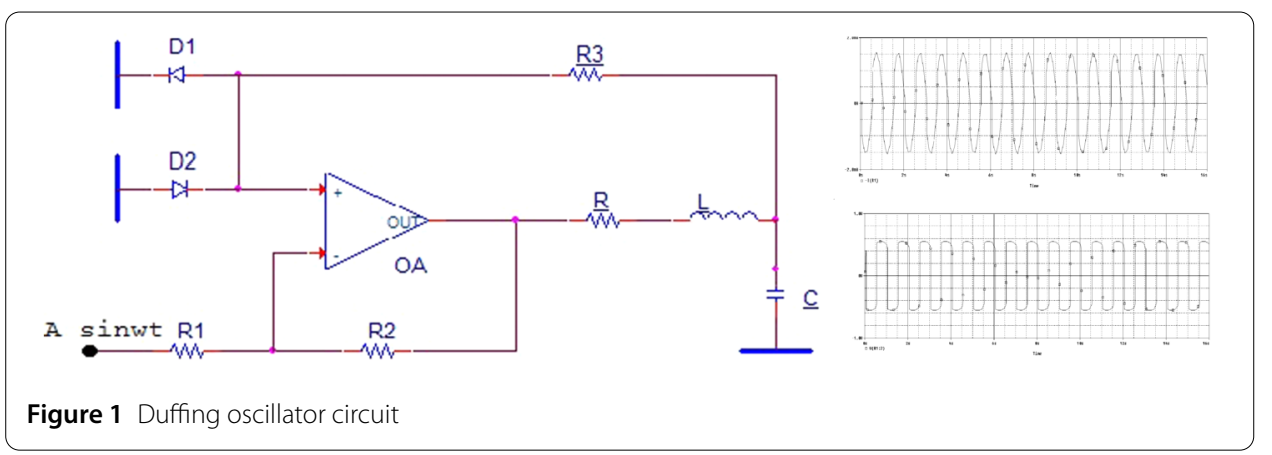

where damping is controlled by $\theta$ (undamped if $\theta=0$ ), $\delta x+\eta x^{3}$ denotes the restoring force of the spring, and the amplitude and angular frequency of the driving force are given by $\gamma$ and $\omega$. The equivalent circuit of the Duffing oscillator with variation of current and voltage across the circuit plotted against time is displayed in Fig. 1. The Duffing equation is used in modeling hard spring oscillators (iron core inductor), soft spring oscillators (nonlinear LRC circuit), negative stiffener, and nonharmonic oscillator [5]. The inverted pendulum equation, which is framed from the Duffing equation, is used in rocket propeller, segway, and robotics.

Fractional calculus, which is regarded as 21st century calculus, has its origin during the same period as that of the ordinary calculus. Continuous fractional-order equations prove to be significant in modeling nuclear reactor dynamics, chaotic dynamical systems, chemical kinetics, population dynamics, and circuit theory[6]. Qualitative analysis of the solutions of fractional-order equations representing real-life phenomena plays a predominant role in understanding the nature and behavior of the models $[7,8]$. Intensive interest shown by researchers during this decade toward discrete fractional calculus demands the need for the development of the methods equivalent to the fractional differential equations. This opinion is very much strengthened by the increase in number of researchers involved in the development of the methodology for discrete fractional calculus [9-12].

The works by Anastassiou [13], Atici and Eloe [14-17], Goodrich [18], and Miller and Ross [19] have laid the foundations for the field of discrete fractional calculus. Time-scale calculus unifies the theory of difference equations with that of differential equations, and qualitative properties such as oscillation and nonoscillation of the dynamic equations on discrete time scales were studied in [20-22]. Chen [23,24] was the first author who studied the stability results of the nonlinear fractional difference equations. The response given by Hyers to the question put forth by Ulam during his talk $[25,26]$ was marked as the origin for the study on stability of functional equations. The Ulam stability of integerand arbitrary-order differential equations were established in [27-30]. Recently, boundary value impulsive integrodifferential equations and coupled systems of Hilfer-Hadamardtype fractional equations are considered for discussion of stability in the Hyers-Ulam sense [31, 32]. Ulam stability analysis of nabla fractional equations was carried out in [3335], and in [36], the Ulam-Hyers stability of discrete fractional boundary value problems was studied. Here we consider the discrete-time forced fractional-order Duffing equation without damping. 
Denote $\mathbb{Q}:=[j+2, j+\mathbb{T}] \cap \mathbb{N}_{j+2}$, where $\mathbb{T} \in \mathbb{N}$ and $\mathbb{N}_{j}=\{j, j+1, \ldots\}, j \in \mathbb{R}$. Our main equation has the form

$$
\left\{\begin{array}{l}
\Delta_{*}^{v}[\psi(n)]+\delta \psi(n+v)+\eta(\psi(n+v))^{3}+p(n+v)=0, \\
\quad n \in[0, \mathbb{T}] \cap \mathbb{N}_{2-v}, 1<v \leq 2, \\
\psi(0)=A, \quad \Delta(\psi(0))=B,
\end{array}\right.
$$

where $\Delta_{*}^{v}$ is the Caputo like difference operator, $\delta$ and $\eta$ control the linear stiffness and nonlinearity in restoring force, $p: \mathbb{Q} \rightarrow \mathbb{R}$ is the driving force with $A, B \in \mathbb{R}^{+}$. The restoring force represented by $\delta \psi+\eta(\psi)^{3}$ is vital in determining the nature of the spring to be used in the model. The positive real values of $\delta$ and $\eta$ describe the hardening spring, and $\eta<0$ $(\delta>0)$ denotes soft spring.

The choice of the operator plays a crucial role in developing models arising in physics. Here the construction of the physical model using the Caputo difference operator is preferred over the Riemann-Liouville operator to overcome some limitations of the Riemann-Liouville operator in modeling real-life problems. One of the limitations concern the initial conditions defined for the physical problems. Initial conditions of a Caputotype fractional difference operator are traditional integer-order $\left(\Delta^{k}, k \in \mathbb{N}_{0}\right)$ conditions, whereas for a Riemann-Liouville type operator, they are defined in terms of a fractional sum or difference terms $\left(\Delta^{\alpha}, \alpha \in \mathbb{R}\right)$, which fail to provide physical interpretation for the model.

The plan of the paper is as follows. Section 2 imparts some necessary definitions, lemmas, and an existence result. Section 3 presents the Hyers-Ulam stability followed with Hyers-Ulam Mittag-Leffler stability in Sect. 4. Appropriate examples accompanied with simulation are provided in Sect. 5 .

\section{Mathematical background}

In this section, we provide some fundamental definitions and lemmas and state an existence result used throughout this work.

Definition 2.1 ([16]) The Fractional sum of order $v>0$ for a function $p$ is given by

$$
\Delta^{-v} p(n)=\frac{1}{\Gamma(v)} \sum_{r=j}^{n-v}(n-r-1)^{(v-1)} p(r)
$$

where $p$ is defined for $r=j \bmod (1)$, and $\Delta^{-v} \psi$ is defined for $n=(j+v) \bmod (1)$ and $n^{(v)}=\frac{\Gamma(n+1)}{\Gamma(n-v+1)}$.

Definition 2.2 ([16]) Let $\gamma>0$ and $b-1<\gamma<b$, where $b \in \mathbb{N}_{0}, b=\lceil\gamma\rceil$, and $\lceil\cdot\rceil$ denotes the ceiling of a number. Set $v=b-\gamma$. The Caputo fractional difference of order $\gamma$ is

$$
\begin{aligned}
\Delta_{*}^{\gamma} p(n) & =\Delta^{-\nu}\left(\Delta^{b} p(n)\right) \\
& =\frac{1}{\Gamma(v)} \sum_{r=j}^{n-v}(n-r-1)^{(v-1)}\left(\Delta^{b} p\right)(r), \quad \forall n \in \mathbb{N}_{j+\nu} .
\end{aligned}
$$


Lemma 2.3 ([13]) For noninteger $\gamma>0, b=\lceil\gamma\rceil, v=b-\gamma, p$ defined on $\mathbb{N}_{j}$ with $j \in \mathbb{Z}^{+}$, we have

$$
p(n)=\sum_{m=0}^{b-1} \frac{(n-j)^{(m)}}{m !} \Delta^{m}[p(j)]+\frac{1}{\Gamma(\gamma)} \sum_{r=j+\nu}^{n-\gamma}(n-r-1)^{(\gamma-1)} \Delta_{*}^{\gamma}[p(r)] .
$$

In particular, if $1<\gamma<2$ and $j=0$, then this relation becomes

$$
p(n)=p(0)+n \Delta(p(0))+\frac{1}{\Gamma(\gamma)} \sum_{r=2-\gamma}^{n-\gamma}(n-r-1)^{(\gamma-1)} \Delta_{*}^{\gamma}[p(r)],
$$

where $p$ is defined on $\mathbb{N}_{2}$.

Lemma 2.4 A function $\psi: \mathbb{Q} \rightarrow \mathbb{R}$ is a solution of (1.2) if and only if $\psi$ is a solution of

$$
\psi(n)=A+n B+\frac{1}{\Gamma(v)} \sum_{r=2-v}^{n-v}(n-r-1)^{(v-1)}\left(-p(r+v)-\delta \psi(r+v)-\eta(\psi(r+v))^{3}\right)
$$

where $n \in \mathbb{Q}$.

Proof Let $\psi$ be a solution of (1.2). Then from (2.2) we have

$$
\psi(n)=\psi(0)+n \Delta(\psi(0))+\frac{1}{\Gamma(v)} \sum_{r=2-v}^{n-v}(n-r-1)^{(v-1)} \Delta_{*}^{v}[\psi(r)]
$$

or

$$
\psi(n)=A+n B+\frac{1}{\Gamma(v)} \sum_{r=2-v}^{n-v}(n-r-1)^{(v-1)}\left(-p(r+v)-\delta \psi(r+v)-\eta(\psi(r+v))^{3}\right),
$$

which satisfies (2.3). On the other hand, if $\psi$ is a solution of (2.3), then by comparison of (2.2) and (2.3) we get

$$
\sum_{r=2-v}^{n-v}(n-r-1)^{(v-1)} \Delta_{*}^{v}[\psi(r)]=\sum_{r=2-v}^{n-v}(n-r-1)^{(v-1)}\left(-p(r+v)-\delta \psi(r+v)-\eta(\psi(r+v))^{3}\right),
$$

which takes the form

$$
\begin{aligned}
& \sum_{r=2-v}^{n-v}(n-r-1)^{(v-1)}\left[\Delta_{*}^{v}[\psi(r)]-\left(-p(r+v)-\delta \psi(r+v)-\eta(\psi(r+v))^{3}\right)\right]=0, \\
& \quad n \in \mathbb{Q} .
\end{aligned}
$$

Letting $n=1,2,3, \ldots$ yields

$$
\Delta_{*}^{v}[\psi(n)]+\delta \psi(n+v)+\eta(\psi(n+v))^{3}+p(n+v)=0, \quad n \in \mathbb{Q} .
$$

It is evident that $\psi$ satisfies (1.2). The proof is complete. 
Lemma 2.5 We have

$$
\sum_{r=2-v}^{n-v}(n-r-1)^{(v-1)}=\frac{(n+v-2)^{(v)}}{v} .
$$

Proof For $a, d \in \mathbb{R}$ with $d>a, d>-1$, and $a>-1$, we have

$$
\frac{\Gamma(d+1)}{\Gamma(d-a+1)}=\frac{1}{a+1}\left[\frac{\Gamma(d+2)}{\Gamma(d-a+1)}-\frac{\Gamma(d+1)}{\Gamma(d-a)}\right]
$$

Then

$$
\begin{aligned}
\sum_{r=2-v}^{n-v}(n-r-1)^{(v-1)} & =\sum_{r=2-v}^{n-v} \frac{\Gamma(n-r)}{\Gamma(n-r-v+1)} \\
& =\sum_{r=2-v}^{n-v-1} \frac{\Gamma(n-r)}{\Gamma(n-r-v+1)}+\Gamma(v) \\
& =\sum_{r=2-v}^{n-v-1} \frac{1}{v}\left[\frac{\Gamma(n-r+1)}{\Gamma(n-r-v+1)}-\frac{\Gamma(n-r)}{\Gamma(n-r-v)}\right]+\Gamma(v) \\
& =\frac{1}{v}\left[\frac{\Gamma(n+v-1)}{\Gamma(n-1)}-\Gamma(v+1)\right]+\Gamma(v) \\
& =\frac{(n+v-2)^{(v)}}{v} .
\end{aligned}
$$

To ensure the existence of solution, we consider

$$
F \psi(n)=A+n B+\frac{1}{\Gamma(v)} \sum_{r=2-v}^{n-v}(n-r-1)^{(v-1)}\left(-p(r+v)-\delta \psi(r+v)-\eta(\psi(r+v))^{3}\right) .
$$

To prove the existence, we apply the Krasnoselskii fixed point theorem. Let $W$ be a nonempty, closed, bounded, and convex subset of a Banach space $(S ;\|\cdot\|)$. Suppose that $F_{1}, F_{2}$ map $W$ into $W$ and that

- for all $\psi, \phi \in W, F_{1} \psi+F_{2} \phi \in W$,

- $F_{1}$ is continuous, and $F_{1} W$ is contained in a compact subset of $W$,

- $F_{2}$ is a contraction.

Then there is $z \in W$ such that $z=F_{1} z+F_{2} z$.

We define $W:=\{y \in C(\mathbb{Z} ; \mathbb{R}),\|y\| \leq K\}$, where $C(\mathbb{Z} ; \mathbb{R})$ denotes the set of continuous functions from $\mathbb{Z}$ to $\mathbb{R}$, and $F=F_{1}+F_{2}$, where

$$
F_{1} \psi(n)=A+n B
$$

and

$$
F_{2} \psi(n)=\frac{1}{\Gamma(v)} \sum_{r=2-v}^{n-v}(n-r-1)^{(v-1)}\left(-p(r+v)-\delta \psi(r+v)-\eta(\psi(r+v))^{3}\right) .
$$


Theorem 2.6 Problem (1.2) has a solution in the set $W$, provided that

$$
A+B \mathbb{T}+\frac{1}{\Gamma(v)}(\mathbb{T}+v-1)^{v-1}(\mathbb{T}-2)\left(\|p\|+\delta K+\eta K^{3}\right) \leq K,
$$

where $\|p\|=\sup _{n \in \mathbb{Q}}|p(n)|$. Moreover, the solution is unique if

$$
\frac{1}{\Gamma(v)}(\mathbb{T}+v-1)^{v-1}(\mathbb{T}-2)\left(\delta+\eta 3 K^{2}\right) \leq 1
$$

Proof First, we can easily check that $\left|F_{1} \psi(n)\right| \leq A+B \mathbb{T}$. On the other hand, we compute

$$
\begin{aligned}
\left|F_{2} \psi(n)\right| & \leq \frac{1}{\Gamma(v)} \sum_{r=2-v}^{n-v}|(n-r-1)|^{(v-1)}\left(|p(r+v)|+\delta|\psi(r+v)|+\eta|\psi(r+v)|^{3}\right) \\
& \leq \frac{1}{\Gamma(v)} \sum_{r=2-v}^{n-v}|(n-r-1)|^{(v-1)}\left(\|p\|+\delta K+\eta K^{3}\right) \\
& \leq \frac{1}{\Gamma(v)}(\mathbb{T}+v-1)^{v-1}(\mathbb{T}-2)\left(\|p\|+\delta K+\eta K^{3}\right) \\
& :=W^{*} .
\end{aligned}
$$

We can choose the constants $\delta, \eta, \mathbb{T}$ such that $A+B \mathbb{T}+W^{*} \leq K$. For such a choice, $\mid F_{1} \psi+$ $F_{2} \phi \mid \leq K$, and hence $F_{1} \psi+F_{2} \phi \in W$ for $\psi, \phi \in W$.

The continuity of $F_{1}$ is easy to check as it is just a function of $n$. Besides, the set $F_{1} W$ is bounded. Thus $F_{1} W$ is contained in a compact subset of $W$.

Now we check the contractivity of $F_{2}$. For $y_{1}, y_{2} \in W$, we have

$$
\begin{aligned}
& \left\|F_{2} y_{1}-F_{2} y_{2}\right\| \\
& \quad \leq \frac{1}{\Gamma(v)} \sum_{r=2-v}^{n-v}(n-r-1)^{(v-1)}\left(\delta\left|y_{1}(r+v)-y_{2}(r+v)\right|+\eta\left|\left(y_{1}(r+v)\right)^{3}-\left(y_{2}(r+v)\right)^{3}\right|\right) \\
& \leq \frac{1}{\Gamma(v)} \sum_{r=2-v}^{n-v}(n-r-1)^{(v-1)}\left(\delta\left|y_{1}(r+v)-y_{2}(r+v)\right|+\eta 3 K^{2}\left|y_{1}(r+v)-y_{2}(r+v)\right|\right) \\
& \quad \leq \frac{1}{\Gamma(v)} \sum_{r=2-v}^{n-v}(n-r-1)^{(v-1)}\left(\delta+\eta 3 K^{2}\right)\left|y_{1}(r+v)-y_{2}(r+v)\right| \\
& \leq \frac{1}{\Gamma(v)} \sum_{r=2-v}^{n-v}(n-r-1)^{(v-1)}\left(\delta+\eta 3 K^{2}\right)\left\|y_{1}-y_{2}\right\| \\
& \leq \frac{1}{\Gamma(v)}(\mathbb{T}+v-1)^{v-1}(\mathbb{T}-2)\left(\delta+\eta 3 K^{2}\right)\left\|y_{1}-y_{2}\right\| \\
& \leq K^{*}\left\|y_{1}-y_{2}\right\|
\end{aligned}
$$

where $K^{*}=\frac{1}{\Gamma(v)}(\mathbb{T}+v-1)^{v-1}(\mathbb{T}-2)\left(\delta+\eta 3 K^{2}\right)$. We can choose the parameters so that $K^{*}<1$, so $F_{2}$ is a contraction. Combining the above, the existence of a solution is ensured. 
On the other hand, we can easily see that for $y_{1}, y_{2} \in W$ and $F=F_{1}+F_{2}$, we get

$$
\begin{aligned}
& \left\|F y_{1}-F y_{2}\right\| \\
& \quad \leq \frac{1}{\Gamma(v)} \sum_{r=2-v}^{n-v}(n-r-1)^{(v-1)}\left(\delta\left|y_{1}(r+v)-y_{2}(r+v)\right|+\eta\left|\left(y_{1}(r+v)\right)^{3}-\left(y_{2}(r+v)\right)^{3}\right|\right) \\
& \quad \leq \frac{1}{\Gamma(v)} \sum_{r=2-v}^{n-v}(n-r-1)^{(v-1)}\left(\delta\left|y_{1}(r+v)-y_{2}(r+v)\right|+\eta 3 L^{2}\left|y_{1}(r+v)-y_{2}(r+v)\right|\right) \\
& \quad \leq \frac{1}{\Gamma(v)} \sum_{r=2-v}^{n-v}(n-r-1)^{(v-1)}\left(\delta+\eta 3 L^{2}\right)\left|y_{1}(r+v)-y_{2}(r+v)\right| \\
& \leq \frac{1}{\Gamma(v)} \sum_{r=2-v}^{n-v}(n-r-1)^{(v-1)}\left(\delta+\eta 3 L^{2}\right)\left\|y_{1}-y_{2}\right\| \\
& \leq \frac{1}{\Gamma(v)}(\mathbb{T}+v-1)^{v-1}(\mathbb{T}-2)\left(\delta+\eta 3 L^{2}\right)\left\|y_{1}-y_{2}\right\| \\
& \leq K^{*}\left\|y_{1}-y_{2}\right\| .
\end{aligned}
$$

We can see that for $\mathbb{T} \geq 2, K^{*}$ is nonnegative. Hence under the condition $K^{*}=\frac{1}{\Gamma(v)}(\mathbb{T}+$ $v-1)^{v-1}(\mathbb{T}-2)\left(\delta+\eta 3 L^{2}\right) \leq 1$, the mapping $F$ is a contraction. Applying the Banach fixed point theorem, the existence and uniqueness of solution is ensured. We can see that we do not need any additional assumptions for uniqueness.

\section{Hyers-Ulam stability}

This section provides results on the Hyers-Ulam stability of (1.2).

For $\psi \in W$, define the norm $\|\psi\|=\sup _{n \in \mathbb{Q}}|\psi(n)|$.

Definition 3.1 ([35]) The discrete fractional initial value problem (1.2) is Hyers-Ulam stable if there exists $\mathbb{U}>0$ such that for any $\epsilon>0, \phi \in \mathbb{R}$ satisfies

$$
\left|\Delta_{*}^{v}[\phi(n)]+\delta \phi(n+v)+\eta(\phi(n+v))^{3}+p(n+v)\right| \leq \epsilon, \quad n \in \mathbb{Q},
$$

with $\phi(0)=A, \Delta(\phi(0))=B$. Then there is a solution $\psi(n)$ of $(1.2)$ such that $|\phi(n)-\psi(n)| \leq$ $\mathbb{U} \epsilon$.

Remark 3.2 A function $\phi \in \mathbb{R}$ solves (3.1) if and only if there exists $h: \mathbb{Q} \times \mathbb{R} \rightarrow \mathbb{R}$ that satisfies

$$
\begin{array}{ll}
\text { A1 } & |h(n+v, \phi(n+v))| \leq \epsilon, n \in \mathbb{Q}, \\
\text { A2 } & \Delta_{*}^{v}[\phi(n)]+\delta \phi(n+v)+\eta(\phi(n+v))^{3}+p(n+v)=h(n+v, \phi(n+v)) .
\end{array}
$$

Lemma 3.3 If $\phi$ solves (3.1), then

$$
\begin{aligned}
& \left|\phi(n)-A-n B+\frac{1}{\Gamma(v)} \sum_{r=2-v}^{n-v}(n-r-1)^{(v-1)}\left(\delta \phi(r+v)+\eta(\phi(r+v))^{3}+p(r+v)\right)\right| \\
& \quad \leq \epsilon \frac{(\mathbb{T}+v-2)^{(v)}}{\Gamma(v+1)}
\end{aligned}
$$

for $n \in \mathbb{Q}$. 
Proof If $\phi$ solves (3.1), then by Remark (3.2) and (2.2) the solution to (A2) satisfies

$$
\begin{aligned}
\phi(n)= & A+n B+\frac{1}{\Gamma(v)} \sum_{r=2-v}^{n-v}(n-r-1)^{(v-1)}(h(r+v, \phi(r+v)) \\
& \left.-p(r+v)-\delta \phi(r+v)-\eta(\phi(r+v))^{3}\right)
\end{aligned}
$$

for $n \in \mathbb{Q}$. Hence

$$
\begin{aligned}
& \left|\phi(n)-A-n B-\Delta^{-v}\left(-p(n+v)-\delta \phi(n+v)-\eta(\phi(n+v))^{3}\right)\right| \\
& =\left|\Delta^{-v} h(n+v, \phi(n+v))\right| \\
& \quad \leq \frac{1}{\Gamma(v)} \sum_{r=2-v}^{n-v}(n-r-1)^{(v-1)}|h(r+v, \phi(r+v))| \\
& \quad \leq \epsilon \frac{1}{\Gamma(v)} \sum_{r=2-v}^{n-v}(n-r-1)^{(v-1)} \\
& \quad \leq \epsilon \frac{(\mathbb{T}+v-2)^{(v)}}{\Gamma(v+1)} .
\end{aligned}
$$

The proof is complete.

We make the following assumptions before proving the stability of (1.2).

(H1) $\sqrt{M}=\max _{n \in \mathbb{Q}}|\psi(n)|$.

(H2) The function $E(n+v, \psi)=-p(n+v)-\delta \psi-\eta(\psi)^{3}$ is Lipschitz continuous, that is, there exists a constant $L>0$ such that for all $\psi, \phi \in \mathbb{R}$ and $n \in \mathbb{Q}$,

$$
|E(n, \psi)-E(n, \phi)| \leq L|\psi-\phi|
$$

where $L=\delta+3 M \eta$.

Theorem 3.4 Assume that (H2) holds. Let $\phi \in \mathbb{R}$ solve (3.1) for some $\epsilon>0$, and let $\psi \in \mathbb{R}$ be the solution of

$$
\left\{\begin{array}{l}
\Delta_{*}^{v}[\psi(n)]+\delta \psi(n+v)+\eta(\psi(n+v))^{3}+p(n+v)=0, \\
\quad n \in[0, \mathbb{T}] \cap \mathbb{N}_{2-v}, 1<v \leq 2, \quad \Delta(\psi(0))=\Delta(\phi(0)) .
\end{array}\right.
$$

Then (1.2) is Hyers-Ulam stable, provided that

$$
\Gamma(\mathbb{T}+v-1)[\delta+3 M \eta]<\Gamma(v+1) \Gamma(\mathbb{T}-1) .
$$

Proof It is clear from Lemma (2.4) that the solution $\psi$ of (3.4) satisfies

$$
\begin{aligned}
\psi(n)= & \phi(0)+n \Delta(\phi(0)) \\
& +\frac{1}{\Gamma(v)} \sum_{r=2-v}^{n-v}(n-r-1)^{(v-1)}\left(-p(r+v)-\delta \psi(r+v)-\eta(\psi(r+v))^{3}\right), \quad n \in \mathbb{Q} .
\end{aligned}
$$


Therefore

$$
\begin{aligned}
\mid \phi(n) & -\psi(n) \mid \\
= & \left|\phi(n)-\phi(0)-n \Delta(\phi(0))-\Delta^{-v}\left(-p(n+v)-\delta \psi(n+v)-\eta(\psi(n+v))^{3}\right)\right| \\
= & \mid \phi(n)-\phi(0)-n \Delta(\phi(0))-\Delta^{-v}\left(-p(n+v)-\delta \phi(n+v)-\eta(\phi(n+v))^{3}\right) \\
& -\Delta^{-v}\left(-p(n+v)-\delta \psi(n+v)-\eta(\psi(n+v))^{3}\right) \\
& +\Delta^{-v}\left(-p(n+v)-\delta \phi(n+v)-\eta(\phi(n+v))^{3}\right) \mid \\
\leq & \left|\phi(n)-\phi(0)-n \Delta(\phi(0))-\Delta^{-v}\left(-p(n+v)-\delta \phi(n+v)-\eta(\phi(n+v))^{3}\right)\right| \\
& +\Delta^{-v}\left(\delta|\phi(n+v)-\psi(n+v)|+\eta\left|\phi(n+v)^{3}-\psi(n+v)^{3}\right|\right) \\
\leq & \epsilon \frac{(\mathbb{T}+v-2)^{(v)}}{\Gamma(v+1)}+(\delta+3 M \eta) \Delta^{-v}|\phi(n+v)-\psi(n+v)| .
\end{aligned}
$$

Using $\|\psi\|=\sup _{n \in \mathbb{Q}}|\psi(n)|$, we have

$$
\begin{aligned}
& \|\phi-\psi\| \leq \epsilon \frac{(\mathbb{T}+v-2)^{(v)}}{\Gamma(v+1)}+(\delta+3 M \eta) \frac{(\mathbb{T}+v-2)^{(v)}}{\Gamma(v+1)}\|\phi-\psi\|, \\
& \|\phi-\psi\| \leq \mathbb{U} \epsilon .
\end{aligned}
$$

Thus (1.2) is Hyers-Ulam stable, and the stability constant is $\mathbb{U}=\frac{(\mathbb{T}+v-2)(v)}{\Gamma(v+1)(1-\xi)}$, where $\xi=$ $\frac{(\mathbb{T}+v-2)(v)}{\Gamma(v+1)}(\delta+3 M \eta)$. The proof is complete.

\section{Hyers-Ulam Mittag-Leffler stability}

For the initial value problem (1.2), Hyers-Ulam Mittag-Leffler stability is investigated in this section.

Definition 4.1 ([35]) The initial value problem (1.2) is Hyers-Ulam Mittag-Leffler stable with $F_{v}(\lambda, n)$ if there exists $\mathbb{V}>0$ with the following property:

For every $\epsilon>0, \phi(n) \in \mathbb{R}$ satisfies the inequality

$$
\left|\Delta_{*}^{v}[\phi(n)]+\delta \phi(n+v)+\eta(\phi(n+v))^{3}+p(n+v)\right| \leq F_{v}(\lambda, n) \epsilon, \quad n \in \mathbb{Q},
$$

with $\phi(0)=A, \Delta(\phi(0))=B$. Then there exists a solution $\psi(n)$ of (1.2) such that $\mid \phi(n)-$ $\psi(n) \mid \leq \mathbb{V} \in F_{v}(\lambda, n)$, where $F_{v}(\lambda, n)$ is the discrete Mittag-Leffler function.

Remark 4.2 A function $\phi \in \mathbb{R}$ solves (3.1) if and only if there exists $\chi: \mathbb{Q} \times \mathbb{R} \rightarrow \mathbb{R}$ that satisfies

B1 $|\chi(n+v, \phi(n+v))| \leq \epsilon F_{v}(\lambda, n), n \in \mathbb{Q}$,

B2 $\Delta_{*}^{v}[\phi(n)]+\delta \phi(n+v)+\eta(\phi(n+v))^{3}+p(n+v)=\chi(n+v, \phi(n+v))$. 
Lemma 4.3 If $\phi$ solves (4.1), then

$$
\begin{aligned}
& \left|\phi(n)-A-n B+\frac{1}{\Gamma(v)} \sum_{r=2-v}^{n-v}(n-r-1)^{(v-1)}\left(\delta \phi(r+v)+\eta(\phi(r+v))^{3}+p(r+v)\right)\right| \\
& \quad \leq \frac{\epsilon}{\lambda} F_{v}(\lambda, n)
\end{aligned}
$$

for $n \in \mathbb{Q}$.

Proof If $\phi$ solves (4.1), then using Remark (4.2) and (2.2), we have that the solution to (B2) satisfies

$$
\begin{aligned}
\phi(n)= & \phi(0)+n \Delta(\phi(0)) \\
& +\frac{1}{\Gamma(v)} \sum_{r=2-v}^{n-v}(n-r-1)^{(v-1)}(\chi(r+v, \phi(r+v)) \\
& \left.-p(r+v)-\delta \phi(r+v)-\eta(\phi(r+v))^{3}\right)
\end{aligned}
$$

for $n \in \mathbb{Q}$. Hence we obtain

$$
\begin{aligned}
\left|\phi(n)-\phi(0)-n \Delta(\phi(0))-\Delta^{-v}\left(-p(n+v)-\delta \phi(n+v)-\eta(\phi(n+v))^{3}\right)\right| \\
\quad=\left|\Delta^{-v} \chi(n+v, \phi(n+v))\right| \\
\quad \leq \Delta^{-v}|\chi(n+v, \phi(n+v))| \\
\quad \leq \epsilon \Delta^{-v} F_{v}(\lambda, n) \\
\quad \leq \frac{\epsilon}{\lambda} F_{v}(\lambda, n) .
\end{aligned}
$$

This completes the proof.

Theorem 4.4 Assume that (H2) holds. Let $\phi \in \mathbb{R}$ solve (4.1) for some $\epsilon>0$, and let $\psi \in \mathbb{R}$ be the solution of (3.4). Then (1.2) is Hyers-Ulam Mittag-Leffler stable, provided that

$$
(\mathbb{T}+v-2)^{(v)}[\delta+3 M \eta]<\Gamma(v+1) .
$$

Proof By Lemma (2.4) the solution $\psi$ of (3.4) satisfies

$$
\begin{aligned}
\psi(n)= & \phi(0)+n \Delta(\phi(0)) \\
& +\frac{1}{\Gamma(v)} \sum_{r=2-v}^{n-v}(n-r-1)^{(v-1)}\left(-p(r+v)-\delta \psi(r+v)-\eta(\psi(r+v))^{3}\right)
\end{aligned}
$$

for $n \in \mathbb{Q}$. Therefore

$$
\begin{aligned}
& |\phi(n)-\psi(n)| \\
& \quad=\left|\phi(n)-\phi(0)-n \Delta(\phi(0))-\Delta^{-v}\left(-p(n+v)-\delta \psi(n+v)-\eta(\psi(n+v))^{3}\right)\right| \\
& \quad=\mid \phi(n)-\phi(0)-n \Delta(\phi(0))-\Delta^{-v}\left(-p(n+v)-\delta \phi(n+v)-\eta(\phi(n+v))^{3}\right)
\end{aligned}
$$




$$
\begin{aligned}
& -\Delta^{-v}\left(-p(n+v)-\delta \psi(n+v)-\eta(\psi(n+v))^{3}\right) \\
& +\Delta^{-v}\left(-p(n+v)-\delta \phi(n+v)-\eta(\phi(n+v))^{3}\right) \mid \\
\leq & \left|\phi(n)-\phi(0)-n \Delta(\phi(0))-\Delta^{-v}\left(-p(n+v)-\delta \phi(n+v)-\eta(\phi(n+v))^{3}\right)\right| \\
& +\Delta^{-v}\left(\delta|\phi(n+v)-\psi(n+v)|+\eta\left|\phi(n+v)^{3}-\psi(n+v)^{3}\right|\right) \\
\leq & \frac{\epsilon}{\lambda} F_{v}(\lambda, n)+(\delta+3 M \eta) \Delta^{-v}|\phi(n+v)-\psi(n+v)| .
\end{aligned}
$$

Using $\|\psi\|=\sup _{n \in \mathbb{Q}}|\psi(n)|$, we have

$$
\begin{aligned}
& \|\phi-\psi\| \leq \frac{\epsilon}{\lambda} F_{v}(\lambda, n)+(\delta+3 M \eta) \frac{(\mathbb{T}+v-2)^{(v)}}{\Gamma(v+1)}\|\phi-\psi\|, \\
& \|\phi-\psi\| \leq \mathbb{V} \epsilon F_{v}(\lambda, n) .
\end{aligned}
$$

Thus we can conclude that (1.2) is Hyers-Ulam Mittag-Lefflar stable with $\mathbb{V}=\frac{1}{\lambda(1-\xi)}$, where $\xi=\frac{(\mathbb{T}+v-2)^{(v)}}{\Gamma(v+1)}(\delta+3 M \eta)$. This completes the proof.

\section{Applications}

Springs are elastic in nature, and thus the original shape is regained after it is subject to some stress. They follow Newton's third law of motion: the harder you pull, the harder it hits back. The rapid increase in restoring force of a spring than suggested by Hooke's law remains the main criterion for a spring to be nonlinear and hard. The motion of such hard springs plays a significant role in study and understanding of nonlinear physics. For a hard spring oscillator, the increase in amplitude results in decrease in period.

In this section, we consider a pendulum with its center of mass above its pivot point. One of the common challenges for engineers and researchers is achieving the stability of an inverted pendulum. The applications of the inverted pendulum varies from personal transporters to electronic unicycles. The motion of an inverted pendulum with forcing term $(\tau \sin (\omega t))$ described by Duffing equation is [5]

$$
m \ell^{2} \ddot{x}+b \dot{x}+(k-m g \ell) x+\left(\frac{1}{6} m g \ell\right) x^{3}+\tau \sin (\omega t)=0,
$$

where $b \dot{x}$ is the damping term, $k$ is the stiffness constant, $m$ is the mass provided at the pendulum top by two or more strong magnets, $\ell$ is the effective length of the pendulum, $g$ is the acceleration due to gravity, the maximum torque is denoted by $\tau$, and $\omega$ is the driving frequency.

Example 5.1 Consider the inverted pendulum equation neglecting the damping term $(b=$ 0 ). The discrete fractional version of the equation is given by

$$
\left\{\begin{array}{l}
\Delta_{*}^{1.895}[\psi(n)]+\left(\frac{k}{m \ell^{2}}-\frac{g}{\ell}\right) \psi(n+1.895)+\left(\frac{g}{6 \ell}\right)(\psi(n+1.895))^{3} \\
\quad=-18.5 \sin (n+1.895), \\
\psi(0)=0, \quad \Delta(\psi(0))=1,
\end{array}\right.
$$

where $n \in[0,10] \cap \mathbb{N}_{0.105}$. We will now establish that (5.2) is Hyers-Ulam stable. 
Let the parameters take the values $k=3.15, m=0.8 \mathrm{~kg}, g=9.8 \frac{\mathrm{m}}{\mathrm{s}^{2}}, \ell=510 \mathrm{~mm}$. Then we obtain

$$
\begin{aligned}
& E(n+1.895, \psi(n+1.895)) \\
& \quad=-18.5 \sin (n+1.895)-\left(\frac{k}{m \ell^{2}}-\frac{g}{\ell}\right) \psi(n+1.895)-\left(\frac{g}{6 \ell}\right)(\psi(n+1.895))^{3},
\end{aligned}
$$

which satisfies the assumption $(H 2)$ with $\sqrt{M}=\max _{n \in \mathbb{Q}}|\psi(n)|=1.4149$. Moreover, we get

$$
\begin{aligned}
&|E(n+1.895, \psi(n+1.895))-E(n+1.895, \phi(n+1.895))| \\
&=\mid-18.5 \sin (n+1.895)-\left(\frac{k}{m \ell^{2}}-\frac{g}{\ell}\right) \psi(n+1.895)-\left(\frac{g}{6 \ell}\right)(\psi(n+1.895))^{3} \\
& \quad+18.5 \sin (n+1.895)+\left(\frac{k}{m \ell^{2}}-\frac{g}{\ell}\right) \phi(n+1.895)+\left(\frac{g}{6 \ell}\right)(\phi(n+1.895))^{3} \mid \\
& \leq {\left[\left(\frac{k}{m \ell^{2}}-\frac{g}{\ell}\right)+\left(\frac{M g}{2 \ell}\right)\right]|\psi-\phi| } \\
& \leq 0.0193|\psi-\phi|,
\end{aligned}
$$

where $n \in[0,10] \cap \mathbb{N}_{0}$ and $L=0.0193$. Thus $E$ is Lipschitz continuous for $n \in[0,10] \cap \mathbb{N}_{0}$. It is clear from Theorem 3.4 that $\xi=0.7443<1$.

Let $\epsilon=0.6$ and $\psi(n)=\frac{n^{(2)}}{10^{2}}$ for $n \in[0,10] \cap \mathbb{N}_{0}$. Now we make sure that inequality (3.1) holds. We have

$$
\begin{aligned}
& \mid \Delta_{*}^{1.895}[\psi(n)]+\left(\frac{k}{m \ell^{2}}-\frac{g}{\ell}\right) \psi(n+1.895) \\
& \quad+\left(\frac{g}{6 \ell}\right)(\psi(n+1.895))^{3}+18.5 \sin (n+1.895) \mid \\
& \quad=\left|\Delta^{-0.105} \Delta^{2}\left(\frac{(n)^{(2)}}{100}\right)-17.9503+18.5 \sin (n+1.895)\right| \\
& \quad \leq 0.5764<\epsilon .
\end{aligned}
$$

Theorem 3.4 clearly shows that (5.2) is Hyers-Ulam stable with $\mathbb{U}$ as the stability constant.

The value of $\xi$ given in Theorem 3.4 for different fractional orders with lengths varying from $510 \mathrm{~mm}$ to $610 \mathrm{~mm}$ are tabulated in Table 1 and are plotted in Fig. 2.

Table 1 Illustration of $\xi$ and $v$

\begin{tabular}{lllll}
\hline$v$ & $\xi$ & & & \\
\cline { 2 - 5 } & $\ell=510$ & $\ell=550$ & $\ell=590$ & $\ell=610$ \\
\hline 1.09 & 0.2038 & 0.1890 & 0.1762 & 0.1650 \\
1.19 & 0.2430 & 0.2253 & 0.2100 & 0.1967 \\
1.29 & 0.2883 & 0.2673 & 0.2492 & 0.2333 \\
1.39 & 0.3405 & 0.3157 & 0.2943 & 0.2756 \\
1.49 & 0.4006 & 0.3714 & 0.3462 & 0.3242 \\
1.59 & 0.4693 & 0.4352 & 0.4056 & 0.3799 \\
1.69 & 0.5479 & 0.5080 & 0.4735 & 0.4434 \\
1.79 & 0.6373 & 0.5909 & 0.5508 & 0.5158 \\
1.89 & 0.7389 & 0.6851 & 0.6386 & 0.5981 \\
1.99 & 0.8540 & 0.7918 & 0.7381 & 0.6912 \\
\hline
\end{tabular}




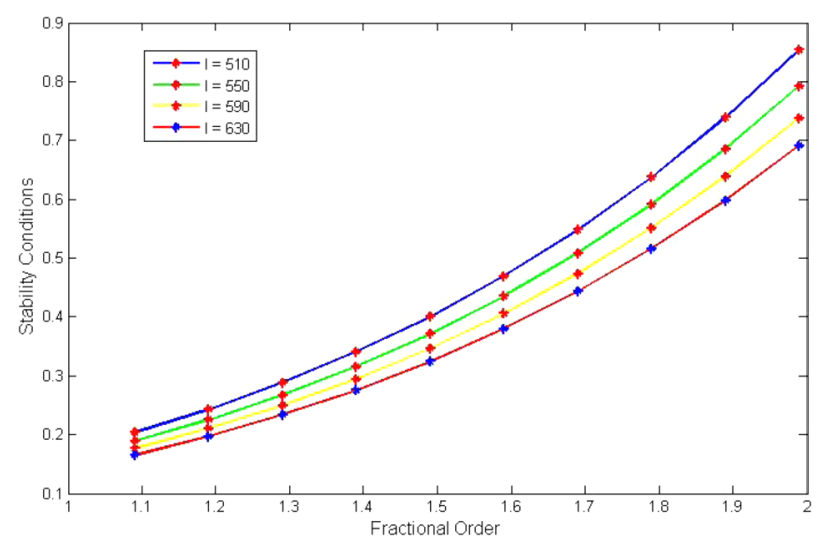

Figure $2 v$ versus $\xi$

Example 5.2 We consider the forced simple harmonic motion equation of discrete-time fractional order. With $v=1.5, \delta=0.01$, and $p(n+v)=0.2 \cos (n+v)$ and neglecting $\eta$ from (1.2), we arrive at

$$
\left\{\begin{array}{l}
\Delta_{*}^{1.5}[\psi(n)]+0.01 \psi(n+1.5)+0.2 \cos (n+1.5)=0, \\
\psi(0)=0, \quad \Delta(\psi(0))=1,
\end{array}\right.
$$

where $n \in[0,13] \cap N_{0.5}$. We will prove the Hyers-Ulam stability of (5.3). Straightforward calculations show that

$$
E(n+1.5, \psi(n+1.5))=0.01 \psi(n+1.5)+0.2 \cos (n+1.5)
$$

is Lipschitz continuous with $L=0.01$. The value of $\xi$ in Theorem 3.4 is $0.3224<1$.

We now ensure that inequality (3.1) holds. Let $\epsilon=0.72$ and $\psi(n)=\frac{n^{(2)}}{20}$ for $n \in[0,13] \cap$ $\mathbb{N}_{0}$. Then

$$
\begin{aligned}
& \left|\Delta_{*}^{1.5}[\psi(n)]+0.01 \psi(n+1.5)+0.2 \cos (n+1.5)\right| \\
& \quad=\left|\Delta^{-0.5} \Delta^{2}\left(\frac{(n)^{(2)}}{20}\right)+0.0979+0.2 \cos (n+1.5)\right| \\
& \quad \leq 0.7008<\epsilon .
\end{aligned}
$$

Thus (3.1) holds, and Theorem 3.4 confirms the Hyers-Ulam stability of (5.3) with constant $\mathbb{U}$.

\section{Conclusion}

Following the trend in investigating equations of fractional order, we consider a discrete fractional form of Duffing equation with forcing term. We accommodate the newly established discrete fractional calculus to determine sufficient conditions for the existence, Hyers-Ulam, stability, and Hyers-Ulam Mittag-Leffler stability for the addressed equation. We analyze practical examples describing the undamped inverted pendulum and forced simple harmonic case as applications of the theoretical results. Stability conditions 
are obtained numerically for different lengths of the pendulum and the values are thus tabulated and represented graphically. We believe that results of this paper are of utmost importance for audience engaged in studying stability of mathematical models describing real physio-electrical phenomena.

\section{Acknowledgements \\ J. Alzabut would like to thank Prince Sultan University for funding this work through research group Nonlinear Analysis Methods in Applied Mathematics (NAMAM) group number RG-DES-2017-01-17.}

Funding

Not applicable.

\section{Availability of data and materials}

Not applicable.

\section{Competing interests}

The authors declare that they have no competing interests.

\section{Authors' contributions}

All authors contributed equally and significantly to this paper. All authors have read and approved the final version of the manuscript.

\section{Author details}

${ }^{1}$ Department of Mathematics, Sacred Heart College, Tirupattur-635601, Tamil Nadu, India. ${ }^{2}$ Department of Mathematics and Computer Sciences, Çankaya University, 06530 Balgat, Ankara, Turkey. ${ }^{3}$ Institute of Space Sciences,

Magurele-Bucharest, Romania. ${ }^{4}$ Department of Mathematics and General Sciences, Prince Sultan University,

Riyadh-11586, Saudi Arabia. ${ }^{5}$ School of Basic Sciences, Indian Institute of Technology Mandi, Kamand, H.P., 175005, India.

\section{Publisher's Note}

Springer Nature remains neutral with regard to jurisdictional claims in published maps and institutional affiliations.

Received: 18 May 2020 Accepted: 20 August 2020 Published online: 01 September 2020

\section{References}

1. Strutt [Lord Rayleigh], J.W.: Theory of Sound, 1. Dover, New York (1877) Re-issued 1945

2. Van der Pol, B.: On "relaxation-oscillations". Philos. Mag. 2(7), 978-992 (1926)

3. Van der Pol, B., Van der Mark, J.: The heartbeat considered as a relaxation oscillation, and an electrical model of the heart. Philos. Mag. 6, 763-775 (1928)

4. Duffing, G.: Erzwungene Schwingungen bei veränderlicher Eigenfrequenz und ihre technische Bedeutung Vieweg, Braunschweig (1918) (German), 41-42

5. Enns, R.H., Mcguire, G.C.: Nonlinear Physics with Mathematica for Scientists and Engineers. Birkhäuser, Boston (2001). ISBN: 3-7643-4223-4

6. Debnath, L.: Recent applications of fractional calculus to science and engineering. Int. J. Math. Math. Sci. 2003(54), 3413-3442 (2003)

7. Mondol, A., Gupta, R., Das, S., Dutta, T.: An insight into Newton's cooling law using fractional calculus. J. Appl. Phys. 123, $064901(2018)$

8. Tarasov, V.E.: Fractional Dynamics: Application of Fractional Calculus to Dynamics of Particles, Fields and Media. Springer, New York (2011)

9. Baleanu, D., Wu, G.: Some further results of the Laplace transform for variable-order fractional difference equations. Fract. Calc. Appl. Anal. 22(6), 1641-1654 (2019)

10. Wu, G., Baleanu, D., Huang, L.: Novel Mittag-Leffler stability of linear fractional delay difference equations with impulse. Appl. Math. Lett. 82, 71-78 (2018)

11. Alzabut, J., Abdeljawad, T.: A generalized discrete fractional Gronwall's inequality and its application on the uniqueness of solutions for nonlinear delay fractional difference system. Appl. Anal. Discrete Math. 12, 036 (2018)

12. Alzabut, J., Abdeljawad, T., Baleanu, D.: Nonlinear delay fractional difference equations with applications on discrete fractional Lotka-Volterra competition model. J. Comput. Anal. Appl. 25(5), 889-898 (2018)

13. Anastassiou, G.A.: Discrete fractional calculus and inequalities. http://arxiv.org/abs/0911.3370

14. Atici, F.M., Sengul, S.: Modeling with fractional difference equations. J. Math. Anal. Appl. 369, 1-9 (2010)

15. Atici, F.M., Eloe, P.W.: Two-point boundary value problems for finite fractional difference equations. J. Differ. Equ. Appl. $17,445-456(2011)$

16. Atici, F.M., Eloe, P.W.: Initial value problems in discrete fractional calculus. Proc. Am. Math. Soc. 137, $981-989$ (2009)

17. Atici, F.M., Eloe, P.W.: Discrete fractional calculus with the nabla operator. Electron. J. Qual. Theory Differ. Equ., Spec. Ed. I 2009, 3, 1-12 (2009)

18. Goodrich, C.S., Peterson, A.C.: Discrete Fractional Calculus. Springer, Cham (2015)

19. Miller, K.S., Ross, B.: An Introduction to the Fractional Calculus and Fractional Difference Equations. Wiley, New York (1993)

20. Zhou, Y., He, J.W., Ahmad, B., Alsaedi, A.: Necessary and sufficient conditions for oscillation of fourth order dynamic equations on time scales. Adv. Differ. Equ. 2019, 308 (2019) 
21. Zhou, Y., Alsaedi, A., Ahmad, B.: Oscillation and nonoscillation theorems of neutral dynamic equations on time scales. Adv. Differ. Equ. 2019, 404 (2019)

22. Zhou, Y., Ahmad, B., Alsaedi, A.: Necessary and sufficient conditions for oscillation of second-order dynamic equations on time scales. Math. Methods Appl. Sci. 42, 4488-4497 (2019). https://doi.org/10.1002/mma.5672

23. Chen, F.: Fixed points and asymptotic stability of nonlinear fractional difference equations. Electron. J. Qual. Theory Differ. Equ. 2011, 39, 1-18 (2011)

24. Chen, F., Liu, Z:: Asymptotic stability results for nonlinear fractional difference equations. J. Appl. Math. 2012, Article ID $879657(2012)$

25. Hyers, D.H.: On the stability of the linear functional equation. Proc. Natl. Acad. Sci. USA 27, 222-224 (1941)

26. Ulam, S.: Problems in Modern Mathematics. Wiley, New York (1964)

27. Niazi, A.U.K., Wei, J., Rehman, M.U., Denghao, P.: Ulam-Hyers-Mittag-Leffler stability of nonlinear fractional neutral differential equations. Sb. Math. 209, 1337-1350 (2018)

28. Ibrahim, R.W.: Generalized Ulam-Hyers stability for fractional differential equations. Int. J. Math. 23(5), 1250056 (2012)

29. Wang, J.R., Zhang, Y.: Ulam-Hyers-Mittag-Leffler stability of fractional-order delay differential equations. Optimization 63(8), 1181-1190 (2014)

30. Ahmad, M., Zada, A., Alzabut, J.: Stability analysis for a nonlinear coupled implicit switched singular fractional differential system with p-Laplacian. Adv. Differ. Equ. 2019, 436 (2019)

31. Ahmad, M., Zada, A., Alzabut, J.: Hyers-Ulam stability of a coupled system of fractional differential equations of Hilfer-Hadamard type. Demonstr. Math. 52, 283-295 (2019). https://doi.org/10.1515/dema-2019-0024

32. Zada, A., Alzabut, J., Waheed, H., Popa, I.: Ulam-Hyers stability of impulsive integrodifferential equations with Riemann-Liouville boundary conditions. Adv. Differ. Equ. 2020, 64 (2020). https://doi.org/10.1186/s13662-020-2534-1

33. Chen, C., Jia, B., Liu, X., Erbe, L.: Existence and uniqueness theorem of the solution to a class of nonlinear nabla fractional difference system with a time delay. Mediterr. J. Math. 15(6), Art. 212, 12 (2018)

34. Jonnalagadda, J.M.: Hyers-Ulam stability of fractional nabla difference equations. Int. J. Anal. 2016, Art. ID 7265307, 5 (2016)

35. Chen, C., Bohner, M., Jia, B.: Ulam-Hyers stability of Caputo fractional difference equations. Math. Methods Appl. Sci. 42(18), 7461-7470 (2019). https://doi.org/10.1002/mma.5869

36. Chen, F., Zhou, Y.: Existence of Ulam stability of solutions for discrete fractional boundary value problem. Discrete Dyn. Nat. Soc. 2013, Article ID 459161 (2013)

\section{Submit your manuscript to a SpringerOpen ${ }^{\circ}$ journal and benefit from:}

- Convenient online submission

- Rigorous peer review

- Open access: articles freely available online

- High visibility within the field

- Retaining the copyright to your article

Submit your next manuscript at $\gg$ springeropen.com 